\title{
The ITER Neutral Beam Test Facility toward SPIDER operation
}

V. Toigo ${ }^{1}$, S. Dal Bello ${ }^{1}$, E. Gaio ${ }^{1}$, A. Luchetta $^{1}$, R. Pasqualotto ${ }^{1}$, P. Zaccaria ${ }^{1}$, M. Bigi ${ }^{1}$, G. Chitarin $^{1}$, D. Marcuzzi ${ }^{1}$, N. Pomaro ${ }^{1}$, G. Serianni ${ }^{1}$, P. Agostinetti ${ }^{1}$, M. Agostini ${ }^{1}$, V. Antoni ${ }^{1}$, D. Aprile ${ }^{1}$, C. Baltador ${ }^{1}$, M. Barbisan ${ }^{1}$, M. Battistella ${ }^{1}$, M. Boldrin ${ }^{1}$, M. Brombin ${ }^{1}$, M. Dalla Palma $^{1}$, A. De Lorenzi ${ }^{1}$, R. Delogu ${ }^{1}$, M. De Muri ${ }^{1}$, F. Fellin ${ }^{1}$, A. Ferro ${ }^{1}$, G. Gambetta ${ }^{1}$, L. Grando $^{1}$, P. Jain ${ }^{1}$, A. Maistrello ${ }^{1}$, G. Manduchi ${ }^{1}$, N. Marconato ${ }^{1}$, M. Pavei ${ }^{1}$, S. Peruzzo ${ }^{1}$, N. Pilan $^{1}$, A. Pimazzoni ${ }^{1}$, R. Piovan ${ }^{1}$, M. Recchia ${ }^{1}$, A. Rizzolo ${ }^{1}$, E. Sartori ${ }^{1}$, M. Siragusa ${ }^{1}$, E. Spada $^{1}$, S. Spagnolo ${ }^{1}$, M. Spolaore ${ }^{1}$, C. Taliercio ${ }^{1}$, M. Valente ${ }^{1}$, P. Veltri ${ }^{1}$, A. Zamengo ${ }^{1}$, B. Zaniol $^{1}$, L. Zanotto ${ }^{1}$, M. Zaupa ${ }^{1}$

D. Boilson ${ }^{2}$, J. Graceffa ${ }^{2}$, L. Svensson ${ }^{2}$, B. Schunke ${ }^{2}$, H. Decamps ${ }^{2}$, M. Urbani ${ }^{2}$, M. Kushwah ${ }^{2}$, J. Chareyre ${ }^{2}$, M. Singh ${ }^{2}$

T. Bonicelli ${ }^{3}$, G. Agarici ${ }^{3}$, A. Masiello ${ }^{3}$, F. Paolucci ${ }^{3}$, M. Simon ${ }^{3}$, L Bailly-Maitre ${ }^{3}$,

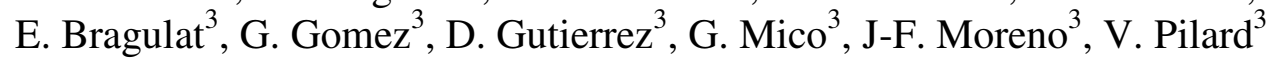
A. Chakraborty ${ }^{4}$,
U. Baruah ${ }^{4}$,
C. Rotti ${ }^{4}$,
H. Patel ${ }^{4}$, M.V. Nagaraju ${ }^{4}$, N.P. Singh ${ }^{4}$,

A. Patel ${ }^{4}$, H. Dhola ${ }^{4}$,

U. Fantz ${ }^{5}$, M. Fröschle ${ }^{5}$, B. Heinemann ${ }^{5}$, W. Kraus ${ }^{5}$, R. Nocentini ${ }^{5}$, R. Riedl ${ }^{5}$, L. Schiesko ${ }^{5}$, C. Wimmer ${ }^{5}$, D. Wünderlich ${ }^{5}$

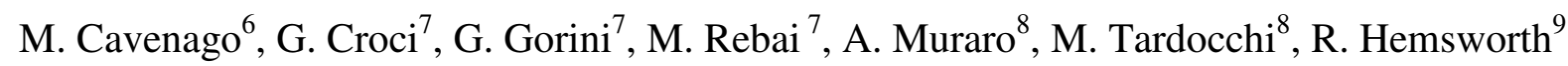

${ }^{1}$ Consorzio RFX, Corso Stati Uniti 4, 35127 Padova, Italy

${ }^{2}$ ITER Organization, Route de Vinon sur Verdon, CS 90 046, 13067 St. Paul Lez Durance Cedex, France

${ }^{3}$ Fusion For Energy, C/o Josep Pla 2, 08019 Barcelona, Spain

4 Institute for Plasma Research, Nr. Indira Bridge, Bhat Village, Gandhinagar, Gujarat 382428, India

${ }^{5}$ IPP, Max-Plank-Institue fur Plasmaphysik, EURATOM Association, D-85748 Garching, Germany

${ }^{6}$ INFN LNL viale dell'Università n. 2, 35020 Legnaro, Italy

${ }^{7}$ Dipartimento di Fisica “G. Occhialini”, Università di Milano-Bicocca, Milano, Italy

${ }^{8}$ Istituto di Fisica del Plasma "P. Caldirola", Milano, Italy

${ }^{9}$ RSH Research Consultants Ltd, 12, Vallon de la Violette, 13820, Ensuès la Redonne, France

E-mail contact of main author: vanni.toigo@igi.cnr.it

\begin{abstract}
SPIDER is one of the two projects of the ITER Neutral Beam Test Facility (NBTF) under construction in Padova, Italy, at Consorzio RFX premises; it will have a $100 \mathrm{keV}$ beam source with a full-size prototype of the radiofrequency (RF) ion source for the ITER Neutral Beam Injector (NBI), designed to operate with pulse length up to $3600 \mathrm{~s}$, featuring ITER-like filter field configuration, caesium oven layout and a wide set of diagnostics. These features will allow reproducing the ion source operation like in ITER as cannot be done in any other existing test facility. SPIDER realization is well advanced and the first operation is expected at the beginning of 2018, with the mission to prove the possibility of achieving the ITER heating and diagnostic NBI ion source requirements and of improving its performance in terms of reliability and availability. This paper mainly focuses on the preparation of the first SPIDER operations: integration and testing of SPIDER components, completion and implementation of diagnostics and control and workout of operation and research plan, based on a staged strategy.
\end{abstract}




\section{Introduction}

In order to achieve thermonuclear-relevant plasma parameters in ITER, the auxiliary heating systems should provide $73 \mathrm{MW}$, out of which $33 \mathrm{MW}$ by two Heating Neutral Beam Injectors (NBI), each designed with beam sources which should operate at $1 \mathrm{MV}, 40 \mathrm{~A}$, for one hour [1]. The unprecedented parameters and the complexity of the NBI systems have led to recognize the need of a dedicated Neutral Beam Test Facility (NBTF) to carry out an international R\&D programme aimed at realizing, testing and optimizing the prototype of the NBIs and at assisting ITER during its operation. This ITER NBTF is under construction [2] in Padova, Italy, at Consorzio RFX premises and hosts two experiments: MITICA, the $1 \mathrm{MeV}$ full-size prototype of the ITER NBI and SPIDER, the test stand for the full-size prototype of the ion source for ITER-NBI and for the diagnostic neutral beam (DNB) for ITER.

The realization of MITICA and SPIDER is proceeding in parallel; the substantial progress of the overall activities is reported in [2]. The ITER Test Facility is realized with the main contribution of the Consorzio RFX, of the European, Japanese and Indian Domestic Agencies (F4E, JADA and INDA), with the support of the ITER Organization and the collaboration of several European laboratories, such as IPP-Garching, KIT-Karlsruhe, CCFE-Culham, CEACadarache. The schedule of the NBTF construction and experiments is strictly aligned with the general timeline of the ITER tokamak experiments, so as to guarantee that sufficient experience on the operation of the prototype source (SPIDER) and of the full Injector (MITICA) will be available before starting the contruction of the Neutral Beam Injectors for ITER.

This paper mainly focuses on the preparation of the SPIDER first operation, expected at the beginning of 2018. SPIDER has a radiofrequency (RF) driven negative ion source, based on a concept developed at IPP Garching [4]; Table I summarizes the requirements to be fulfilled; beam modulation is also prescribed, to test the operation of the source for ITER DNB. Achieving them is very challenging and it will be the result of a common effort, in close collaboration mainly with IPP-Garching, where ELISE is in operation [5], a device half the size of ITER negative ion source also aimed at assessing issues relevant to ITER. However some critical operating conditions can only be tested in SPIDER. In fact, SPIDER is characterized by the same size as ITER NBI ion sources, extraction and acceleration of $\mathrm{H}^{-}$and $\mathrm{D}^{-}$for pulse lengths up to $3600 \mathrm{~s}$, ITER-like filter field configuration and caesium oven layout. Moreover, a wide set of diagnostics is expected to provide design validation and help in operation optimization.

Table 1: SPIDER REQUIREMENTS.

\begin{tabular}{|l|c|c|c|}
\hline & Unit & H & D \\
\hline Beam energy & $\mathrm{keV}$ & 100 & 100 \\
\hline Max beam source filling pressure & $\mathrm{Pa}$ & 0.3 & 0.3 \\
\hline Maximum deviation from uniformity & $\%$ & \pm 10 & \pm 10 \\
\hline Extracted current density & $\mathrm{A} / \mathrm{m}^{2}$ & $>355$ & $>285$ \\
\hline Beam on time & $\mathrm{s}$ & 3600 & 3600 \\
\hline Co-extracted electron fraction $\left(\mathrm{e}^{-} / \mathrm{H}^{-}\right)$and $\left(\mathrm{e}^{-} / \mathrm{D}^{-}\right)$ & & $<0.5$ & $<1$ \\
\hline
\end{tabular}

According to the official ITER schedule, the first plasma will be achieved in 2025 and the pre nuclear phase, requiring the HNB injectors in operation, will take place in 2031. Therefore, several years will be available to exploit the SPIDER mission to prove the possibility of achieving the ITER target requirements in an ion source of the same characteristics and size. 
SPIDER is also aimed at improving the performance in terms of reliability and availability, thus contributing to a significant risk mitigation for the successful operation in ITER.

The preparation of the SPIDER operation is presently on-going; it is a very crucial phase, evolving along three main parallel paths: integration and testing of SPIDER components, completion and implementation of diagnostics and of the Control and Data Acquisition System (CODAS), and preparation of the operation and research plan, based on a staged strategy.

\section{Integration of SPIDER components and complementary research}

The realization of all SPIDER components is well advanced, the next phase on site being the integrated commissioning of the Power Supply (PS) and auxiliary systems. A wide-range testing is planned first on the individual PS components, in remote mode, under the supervision of CODAS. The subsequent phase will consist of a step by step integration of all the PS subsystems to achieve their contemporaneous operation on dummy loads before moving toward the integrated commissioning of SPIDER. In this phase, the attention will not be particularly addressed to the verification of the target parameters of each subsystem in terms of voltage, current, dynamics, already verified during the contractual tests, but mainly on their coordinated operation. At this purpose, special tests requiring specific procedures are planned to verify the correct operation of the subsystems when working together, the coherence of the required sequences, the coordination and reliability of the protection actions, in particular in case of grid breakdowns.

In the last months, first steps of the integrated commissioning have been started; the capability of the High Voltage (HV) Bushing to sustain $120 \mathrm{kVdc}$ for more than $3 \mathrm{~h}$ without breakdowns has already been verified [6] after the development and optimisation of a conditioning procedure. Nevertheless, it is expected that keeping the voltage holding capability over time under the final operational conditions with the Beam Source (BS) installed inside the vessel will require further improvement of the conditioning procedure. Another important target to be achieved was an adequate outgassing rate and vacuum level of

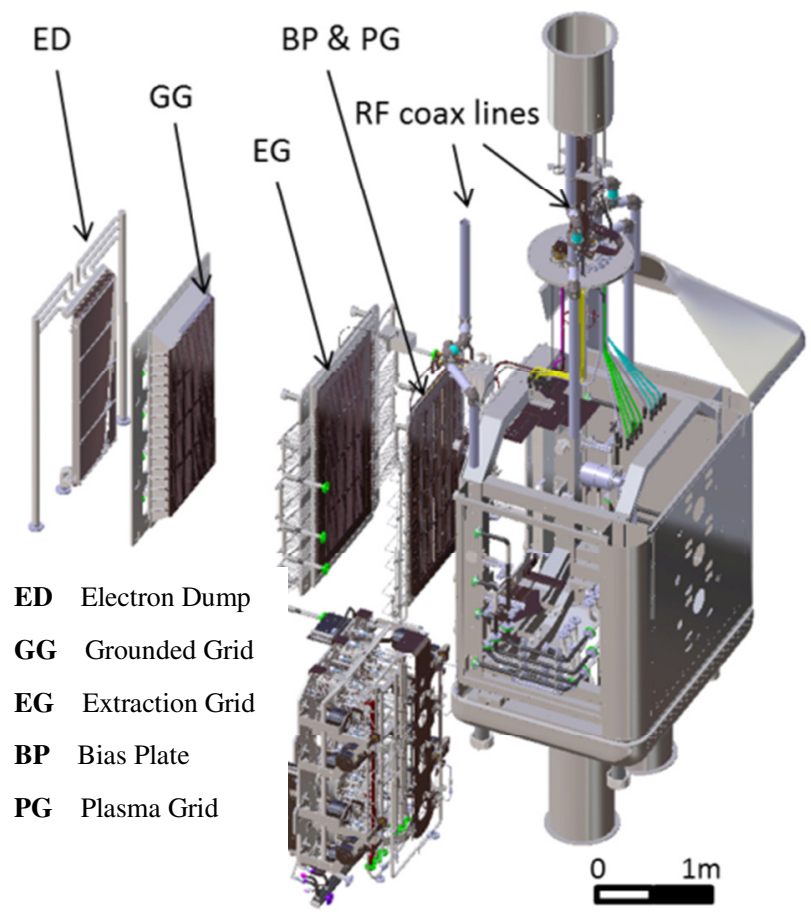

Figure 1 Beam Source 3D exploded view. the SPIDER vessel, necessary to guarantee the correct performance of the ion source. Repeated and accurate cleaning of internal surfaces was carried out and eventually the target outgassing rate was achieved by means of a special baking process carried out for some days, up to $120^{\circ} \mathrm{C}$, using IR lamps in vacuum. As regards gas injection, given the pumping speed installed, tests will be performed to characterize the pressure profile inside the ion source as a function of the injected gas flow. The nominal filling pressure in the source is 0.3 $\mathrm{Pa}$ (with a reference flow of $5 \mathrm{~Pa} \mathrm{~m}^{3} \mathrm{~s}^{-1}$ for $\mathrm{H}_{2}$ ). The gas injection will allow to adjust the pressure from 0.1 to $0.6 \mathrm{~Pa}$. The successful completion of the Beam Source (BS), Figure 1, the most complex component of SPIDER, is the most challenging task. All the parts of the BS have been manufactured and tested in 
factory. Major efforts were required for qualification of manufacturing processes especially for $\mathrm{Cu}$ electrodeposited parts (grids, bias plate and ion source chamber) and heterogeneous $\mathrm{Cu} / \mathrm{SS}$ joints. Difficulties have been encountered in reaching the required quality level especially in terms of extremely tight machining/assembly tolerances, compatibility with vacuum environment and ultrapure water inside the cooling circuits. Assembly of BS in factory is in progress, but some corrective actions were found necessary to recover the proper quality for hydraulic circuit pipes and molybdenum coated surfaces. Actions are on-going to limit within some months the consequent delay in the delivery.

A complementary research toward the SPIDER first operation, in collaboration with IPP, is the development of a caesium oven (Figure 2) capable of assuring vacuum and high temperature $\left(300^{\circ} \mathrm{C}\right)$ compatibility and remote operation.

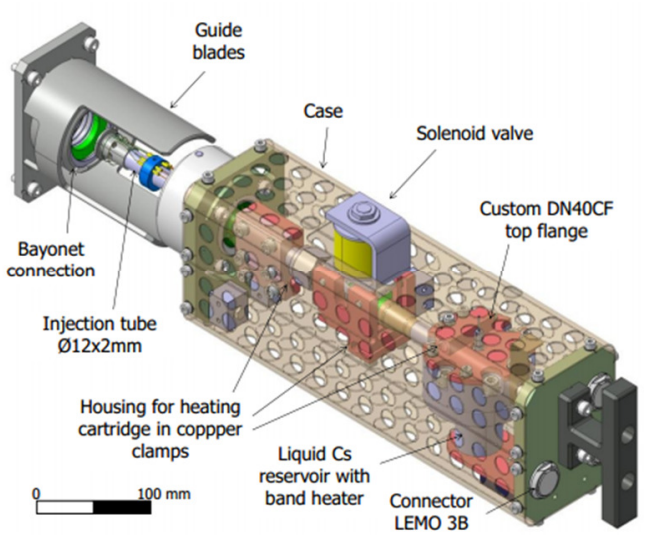

Figure 2 Caesium oven 3D view.

This is an important program, since the production of negative hydrogen (or deuterium) ions inside the sources mainly relies on the generation of ions on caesiated surfaces with a low work function, which enhances the negative ion yield and reduces the coextracted electrons, as verified at ELISE test bed [5]. In the SPIDER beam source fresh caesium will be injected in a controlled way by means of three caesium ovens, installed inside the vacuum vessel. The design is complicated by the high reactivity of caesium, the difficulty in setting a stable (but adjustable) temperature distribution among the parts in vacuum, and the need for a special

customized solenoid valve in vacuum. Before the procurement of the three ovens, a caesium oven test bed is being set up, where a prototype of oven [7] will be tested and characterised inside a vacuum chamber.

The design of suitable Electromagnetic (EM) Shields (Figure 3) for RF drivers required specific studies: copper EM shields are expected to help in reducing the coupling among the BS drivers, thus allowing improvements of the uniformity of the magnetic field inside each driver, as emerged from ELISE operation [5]. The work was mainly addressed to study how the implementation of the EM shields can be performed on the existing BS layout of SPIDER from all the viewpoints: mechanical, electrical and thermohydraulic.

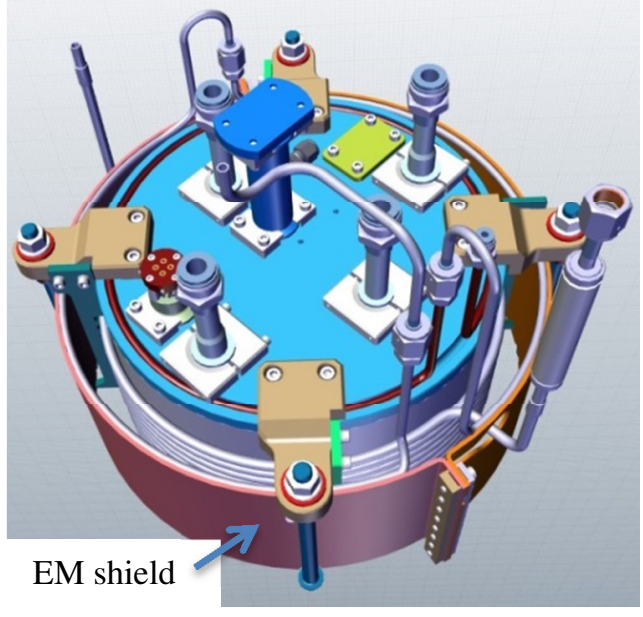

Figure 3 Rf driver with EM shield.

\section{SPIDER Diagnostics}

Since diagnostics in ITER NBIs are essentially limited to thermocouples, due to neutron and gamma radiation and to a limited number of access ports, it is crucial to get as much as possible information from more accessible experiments like MITICA and SPIDER, where diagnostics should demonstrate achievement of the challenging target performance in terms of beam intensity, ratio between coextracted electrons and negative ions, uniformity and divergence over the beam profile for up to one hour pulses. MITICA and SPIDER diagnostics have been designed with this scope [8]. Some of the diagnostics are dedicated to the RF 
source, to characterize the source extraction region, where negative ions are mainly produced and extracted. Others will measure the beam characteristics: the intensity profile, its uniformity and divergence. Information from the two sets of measurements will allow to correlate the physics of the source to the beam characteristics in the actual HNB geometry, and to optimize the operational space of the RF source and of the accelerator. SPIDER is the ideal test stand to perform this investigation, because MITICA will allow much less access and will have a neutron rate intermediate between SPIDER and ITER NBIs. Different methods are used to measure the same parameters, so that they complement and validate each other, thus mitigating the intrinsic limitations of each technique. The measurements will then be more confidently used to validate the physics models of source and beam and to understand what information can be obtained by the limited set of measurements available on ITER NBIs. The suite of diagnostics, shown in Figure 4 and described in [8], [9], includes both techniques well established in other facilities and new methods never used before in this application.

Given the wide variety of implemented techniques and the level of customization needed, requiring a wide range of specialized expertise, the overall development path, from design to

\section{Beam diagnostics:}

Calorimetry and surface thermocouples (beam uniformity, divergence, aiming)

Instrumented calorimeter STRIKE

(beam uniformity over 2D profile \& divergence,

resolution $2 \mathrm{~mm},<10 \mathrm{~s}$ beam pulse)

Beam emission spectroscopy

(beam divergence $\&$ uniformity, stripping losses)

Beam tomography

(beam uniformity over 2D profile,

resolution $1 / 4$ beamlet group)

Neutron imaging

(beam uniformity horiz. profile,

resolution 3-4 cm, D only)

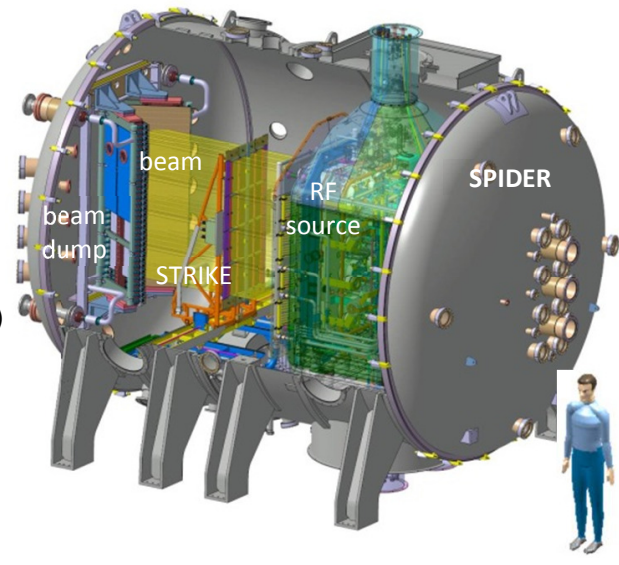

Source diagnostics:

Electrical currents (current balance at power supplies)

Calorimetry and surface

thermocouples

(power load on source components)

Electrostatic probes

(Plasma uniformity, $\mathrm{T}_{\mathrm{e}^{\prime}} \mathrm{n}_{\mathrm{e}}$ )

Source optical emission spectroscopy

( $\mathrm{T}_{\mathrm{e}^{\prime}} \mathrm{n}_{\mathrm{e}^{\prime}}, \mathrm{n}_{\mathrm{H}^{-}}, \mathrm{n}_{\mathrm{Cs}^{\prime}} \mathrm{n}_{\mathrm{H}^{\prime}}$ impurities),

$\operatorname{CRDS}\left(\mathrm{n}_{\mathrm{H}}-\right)$, Laser absorption $\left(\mathrm{n}_{\mathrm{Cs}}\right)$

Figure 4 SPIDER in-vessel view and list of source and beam diagnostics with measured parameters

procurement, installation and commissioning, is managed directly by the hosting laboratory rather than through a contract with industry as for other plants. The design of SPIDER diagnostics is now finalized for most systems and components are being procured, while installation and integration are also starting (Table 2).

Table 2 : STATUS OF DEVELOPMENT OF SPIDER DIAGNOSTICS.

\begin{tabular}{|l|l|}
\hline Diagnostic & Development status \\
\hline Thermocouples & $\begin{array}{l}\text { Layout defined and most components procured; custom } \\
\text { conditioning electronics under test; ready to start installation }\end{array}$ \\
\hline Electrostatic probes & $\begin{array}{l}\text { Custom electronics with full isolation of every channel under } \\
\text { prototyping; probes ready for installation on beam source }\end{array}$ \\
\hline Spectroscopy & $\begin{array}{l}\text { Optical heads, fibers, spectrometers and cameras procured; } \\
\text { filtered detectors under prototyping; ready to start installation; } \\
\text { data analysis tools under test on NIO1 [10] and ELISE }\end{array}$ \\
\hline STRIKE & $\begin{array}{l}\text { Prototype tiles qualified; full set of tiles production starting; in } \\
\text { vacuum support and positioning system under manufacturing; 2D } \\
\text { power profile reconstruction in progress; small scale diagnostic }\end{array}$ \\
\hline
\end{tabular}




\begin{tabular}{|l|l|}
\hline & used on BATMAN [4], [11], NIO1, NIFS test bed [12], [13] \\
\hline Beam Tomography & $\begin{array}{l}\text { Prototype cameras under test; tomographic inversion algorithm } \\
\text { developed }\end{array}$ \\
\hline Neutron imaging & $\begin{array}{l}\text { nGEM imaging detector fully tested; vacuum box for detector } \\
\text { enclosure manufactured }\end{array}$ \\
\hline IR thermography & Microbolometer IR cameras procured \\
\hline Viewports, feedthroughs & Procured and ready for installation \\
\hline software & $\begin{array}{l}\text { MDSplus [14] drivers under development for central } \\
\text { management of acquisition and remotely controlled devices of all } \\
\text { diagnostics }\end{array}$ \\
\hline
\end{tabular}

In the ion source and on the water cooled beam dump, thermocouples (TCs) are used both for calorimetry, fastened to the inlet and outlet pipes of the cooling circuit, and for local heat load estimates, located just at few $\mathrm{mm}$ from the heated surfaces. Source plasma parameters are measured by the combination of optical emission spectroscopy, assisted by a collisional radiative model, and electrostatic probes (Te, ne), together with cavity ring-down spectroscopy for $\mathrm{H}^{-}$density, and laser absorption spectroscopy for atomic caesium density. Spectroscopy measurements distributed over multiple lines-ofsight (LOS) and Langmuir probes

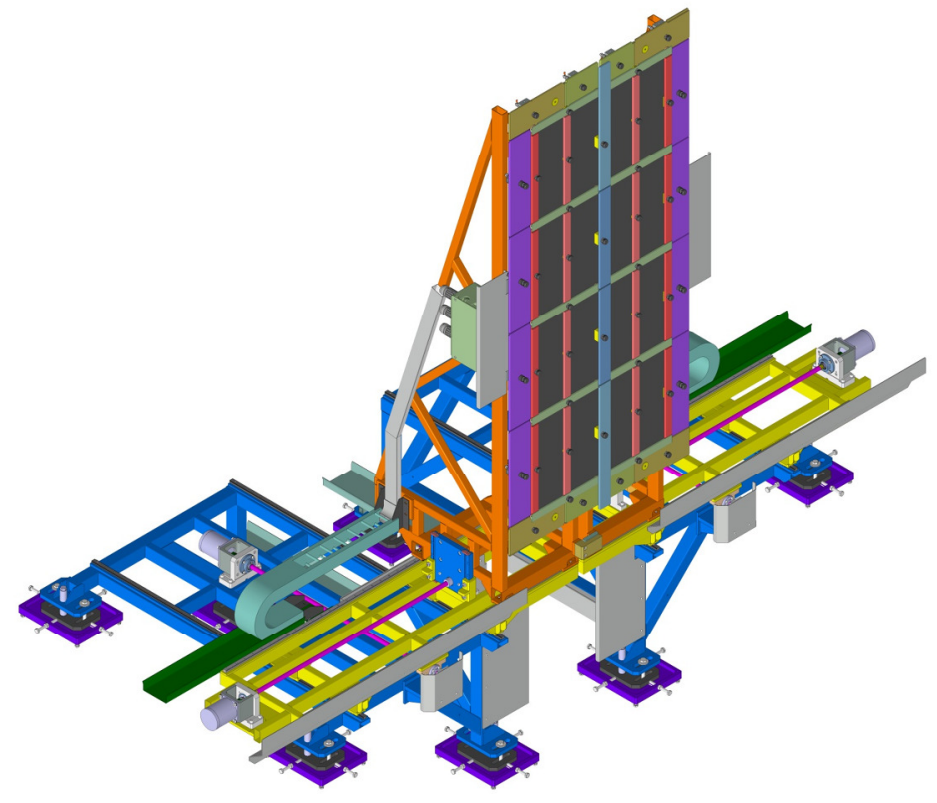

Figure 5 CAD model of STRIKE; front surface hit by the beam is shown

over the Plasma Grid (PG) and Bias Plate shall give information on the spatial distribution of the plasma parameters over the extraction region, investigating drift and non-uniformity of the plasma profile induced by the filter magnetic field and caesium evaporation. Probes have a custom design and can be configured as either single sensors, to measure the ion saturation current, or coupled to a reference electrode to get the whole characteristic curve. An invacuum RF compensation circuit with passive components has been developed and tested, to minimize the noise and distortion induced by the RF environment. Emission spectroscopy measures both time evolution of single spectral lines and of the entire spectrum, and is used to verify the correct ignition of RF drivers and the plasma pollution by impurities, particularly important in the first commissioning phase, and detects the spectrally resolved Balmer series and molecular spectra of hydrogen or deuterium, to extimate density and temperature of all plasma species.

As described in [8], [9], the beam profile uniformity and its divergence are studied with wellassessed techniques like TCs and beam emission spectroscopy (BES), by other methods like visible tomography, and by innovative methods like neutron imaging and the instrumented calorimeter (STRIKE). STRIKE, shown in Figure 5, is the main beam diagnostic in SPIDER 
as it is expected to achieve the spatial resolution of $2 \mathrm{~mm}$ over the entire beam profile, thus validating all other systems, especially those based on LOS integrated measurements like BES and tomography. STRIKE is made of 16 CFC-1D tiles that intercept the entire beam and, thanks to the preferential heat conduction through the tile rather than across it, well reproduce the front heated surface on the back side, whose temperature map can then be recorded by two infrared cameras. Beside beam uniformity, STRIKE can measure its divergence, by positioning the tiles at different distances from the source exit, and the collected beam current, as tiles are insulated from their support. The main limitation is due to the fact that the tiles are inertially cooled and then they can only survive up to 10 s beam pulses at full power, so that it will be remotely positioned out of the beam path for long high-power pulses; on the other hand, STRIKE will be particularly important in the initial operational phase at lower power to

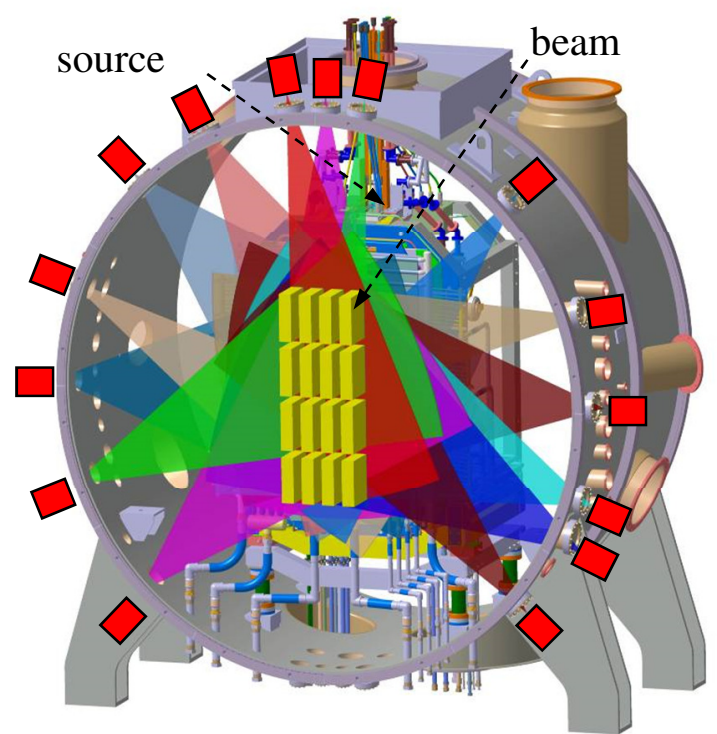

Figure 6 Vertical cross-section of the SPIDER vessel with view of the $4 \times 4$ beamlet groups and 15 linear cameras of the tomographic diagnostic with corresponding fans of lines of sight tune the source and accelerator parameters. For longer pulses the highest spatial resolution is achieved by visible beam tomography, which measures the light emitted by the interaction of the beam with the background gas along about 3000 Lines Of Sight (LOSs), arranged in 15 fans associated to linear cameras (Figure 6). Tomography can measure beam uniformity with a resolution down to a pair of beamlets if suitable regularization is applied in the inversion. The $\mathrm{D}^{-}+$ $\mathrm{D}$ beam intensity profile will be also derived from the map of neutron emission on the beam dump surface, due to fusion reactions between beam deuterons and deuterons previously adsorbed in the dump, for a global yield of $\approx 10^{12} \mathrm{n} / \mathrm{s}$. It is based on Gas Electron Multiplier (GEM) detectors with a cathode that serves also as neutron-proton converter, installed just behind a beam dump panel. It achieves a resolution of about $3 \mathrm{~cm}$, but it can operate only in deuterium. Finally, information on beam intensity map, its uniformity and divergence can be derived by TCs on the water cooled beam dump. Comparing these measurements with the other beam diagnostics will help understanding what information on the beam can be achieved in ITER NBIs, through TC measurements distributed over the beam line components. SPIDER diagnostic prototypes are being operated in other experiments to verify their capabilities and to validate models. As an example, a small scale prototype of STRIKE has proven capable of resolving small-scale structures in the beam profile at operational test beds in IPP Garching, NIFS, Japan and QST, Japan.

\section{Operation and early research Plan}

The preparation of the SPIDER operation and research plan is proceeding in parallel to the integration and testing of SPIDER components and diagnostics. The present scenario involves an integrated commissioning followed by the SPIDER operation.

\section{a. Integrated commissioning}

The integrated commissioning will be performed with the source already installed in the vacuum vessel after a first commissioning of the individual plants, associating the test parameters of the plants in each commissioning phase to the values required for the following 
operational phase. This staged strategy is preferred with respect to the alternative full commissioning of each plant before the start of SPIDER operation, as it represents a faster track towards the experimentation, which would anyway start at reduced performance. The staged commissioning will also reduce the risk of delays in starting experiments; on the other hand, subsequent SPIDER operation with higher parameters of the various plants will require dedicated commissioning phases at a later time.

The integrated commissioning of SPIDER is divided into three phases depending on the atmosphere inside the SPIDER vacuum vessel, which can be air, vacuum, and hydrogen. The first activities will be performed in air to have easy access for measuring voltages and currents without the need of high voltage electrical feedthroughs and of repeatedly opening the vacuum; the voltage holding test of the extractor and accelerator insulators can be carried out within the permissible limits in this testing condition. Specifically, the following steps are planned in air:

- the RF circuits, each one including one power supply, matching network capacitors and two RF drivers, are first tested one by one, for their electrical characterisation and verification of their frequency response

- the RF circuits are tested in pairs and finally all of them are switched on together; RFinduced noise on other components will be assessed

- the magnetic filter field circuit will be powered, while monitoring the voltage drops along the circuit branches; correspondingly, the magnetic field will be measured in relevant positions for an experimental mapping to be compared with numerical computations; the influence of the filter field on the operation of the RF circuits will be assessed

- bias plate, bias circuit and PG bias circuit will be powered and a low current will be applied to the ignition filaments

- the high voltages will be applied to the extraction stage and to the acceleration stage, to check the voltage holding of the insulators and to assess their leakage currents

At the end of these tests, it is expected that the correct operation of the whole system and the voltage holding capabilities of the coolant circuits and of the insulators are verified.

In vacuum, the tests aim to verify the voltage holding capabilities of the various gaps, including those in the RF coils of the drivers. High voltage conditioning of the accelerator grids will be performed by gradually raising the voltage. Some of the tests of other power supplies, already performed in air, will be repeated; the current in the ignition filaments will be raised so that they start emitting a thermionic current.

Table 3 FIRST SPIDER OPERATION PLAN.

\begin{tabular}{|c|c|c|c|c|}
\hline Stage & H/D & RF Power & Extraction & Caesium \\
\hline 1 & $\mathrm{H}$ & $<200 \mathrm{~kW}$ & NO & NO \\
\hline 2 & $\mathrm{H}$ & $<200 \mathrm{~kW}$ & YES & NO \\
\hline 3 & $\mathrm{H}$ & $<400 \mathrm{~kW}$ & YES & NO \\
\hline 4 & $\mathrm{H}$ & $<400 \mathrm{~kW}$ & YES & YES \\
\hline
\end{tabular}

Finally, while injecting hydrogen into the beam source and verifying the pumping capabilities, the voltage holding of extractor and accelerator gaps will be verified in the range 
of expected pressure conditions; the RF circuits will be powered without filament current; then the plasma ignition will be tested and its influence on other plants can be assessed.

\section{b. Early research plan}

The early experimental phase of SPIDER is planned to follow a staged approach [15][16], as summarized in Table 3; the expected duration of early SPIDER operation is shown in Figure 7.

The main results of the first stage should be a characterization of the plasma ignition and ion source operation, without caesium and at low RF power (around $200 \mathrm{~kW}$ ), an assessment of the influence of the filter field on plasma ignition and the ion source uniformity. The dependence of plasma parameters on filter field, bias plate bias and PG bias will be investigated in the vicinity of the Plasma Grid (PG) at different gas pressures; the plasma drifts and non-uniformities in the same region will be verified. The pulse duration will be limited, taking into account in particular the heat load measured at all surfaces and components hit by the beam, including STRIKE.

\begin{tabular}{|c|c|c|c|c|c|c|c|c|c|c|c|c|c|c|c|c|c|c|c|c|c|}
\hline stage & Activity & 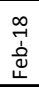 & 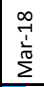 & $\begin{array}{l}\infty \\
\frac{1}{1} \\
\frac{1}{2}\end{array}$ & $\begin{array}{l}\infty \\
\stackrel{1}{1} \\
\frac{1}{\pi} \\
\sum\end{array}$ & 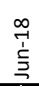 & $\begin{array}{l}\infty \\
\stackrel{1}{\Xi} \\
\frac{1}{3}\end{array}$ & 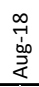 & $\begin{array}{l}\infty \\
\stackrel{\infty}{1} \\
\dot{\alpha} \\
\sim \\
\sim\end{array}$ & $\begin{array}{l}\infty \\
\stackrel{+}{\sharp} \\
\stackrel{\Delta}{\Delta}\end{array}$ & $\begin{array}{l}\infty \\
\text { 1 } \\
\text { 3े } \\
2\end{array}$ & $\begin{array}{l}\infty \\
\stackrel{\sim}{u} \\
\text { ه్ }\end{array}$ & 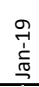 & $\begin{array}{l}0 \\
\overrightarrow{7} \\
\dot{1} \\
\text { ऐ. } \\
\end{array}$ & $\begin{array}{l}9 \\
7 \\
\frac{1}{10} \\
\sum\end{array}$ & $\begin{array}{l}\frac{1}{7} \\
\frac{1}{2}\end{array}$ & 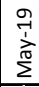 & 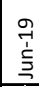 & $\begin{array}{l}\frac{9}{7} \\
\frac{1}{3}\end{array}$ & 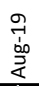 & $\begin{array}{l}\stackrel{9}{1} \\
\frac{1}{2} \\
\stackrel{0}{n}\end{array}$ \\
\hline & characterisation of plasma ignition & & & & & & & & & & & & & & & & & & & & \\
\hline \multirow[t]{4}{*}{1} & characterisation of ion source & & & & & & & & & & & & & & & & & & & & \\
\hline & optimisation of ion source uniformity & & & & & & & & & & & & & & & & & & & & \\
\hline & inspection & & & & & & & & & & & & & & & & & & & & \\
\hline & high voltage holding; accelerator conditioning & & & & & & & & & & & & & & & & & & & & \\
\hline \multirow{5}{*}{2} & first tests of beam extraction and acceleration & & & & & & & & & & & & & & & & & & & & \\
\hline & investigation of beamlet optics and clearance & & & & & & & & & & & & & & & & & & & & \\
\hline & investigation of zigzag beamlet deflection & & & & & & & & & & & & & & & & & & & & \\
\hline & inspection & & & & & & & & & & & & & & & & & & & & \\
\hline & characterisation of beam extraction and acceleration & & & & & & & & & & & & & & & & & & & & \\
\hline \multirow[t]{3}{*}{3} & optimisation of beamlet optics and clearance & & & & & & & & & & & & & & & & & & & & \\
\hline & investigation of zigzag beamlet deflection & & & & & & & & & & & & & & & & & & & & \\
\hline & characterisation of ion source with caesium & & & & & & & & & & & & & & & & & & & & \\
\hline \multirow{4}{*}{4} & characterisation of beam extraction and acceleration & & & & & & & & & & & & & & & & & & & & \\
\hline & optimisation of source and of beamlet optics and clearance & & & & & & & & & & & & & & & & & & & & \\
\hline & assessment and optimisation of caesium consumption & & & & & & & & & & & & & & & & & & & & \\
\hline & inspection & & & & & & & & & & & & & & & & & & & & \\
\hline
\end{tabular}

Figure 7 Planning of the early phase of SPIDER experiments.

After checking that the plasma leaking out of the ion source has not spoilt the voltage holding capabilities of the accelerator and after high-voltage conditioning of the accelerator, a first beam at low current density will be extracted and accelerated, allowing the assessment of the effect of charged particles on voltage holding. In this phase the design of SPIDER extractor and accelerator [17], including the similarities to the design of MITICA accelerator [18], will be validated: extraction and acceleration optics, which combined together determine the final optimal perveance [15], will be studied, including the assessment of beam aiming and beam clearance inside the apertures of the EG. This phase will involve several investigations of the accelerator performance, like the amount of co-extracted electrons, the influence of PG bias, bias plate bias and filter field, and the compensation of the zigzag deflection of beamlets due to the permanent magnets located in the EG. Concerning the last issue, it must be remembered that SPIDER provides the unique chance of comparing in the same machine the magnetic compensation of the zigzag deflection of ions (expected to be independent from the particle energy at first order) and the usually adopted electrostatic compensation (depending on particle energy); for comparison, some rows of beamlets are left without any compensation. Such a comparison is even more important in the first stages of SPIDER operation, when the operating conditions will not be the nominal ones. 
After this stage, the RF power will be progressively raised and the characterisation of the source and of the beam will continue, extending the range of parameters. The operational phase will continue with caesium injection and with the corresponding characterisation of the ion source plasma and of the beam; a first attempt to optimize the beam properties will be performed in view of the ITER requirements, see Table 1: beam uniformity, compensation of zigzag deflection, electron-to-ion ratio; a first assessment of caesium consumption will also ensue. In all stages of Table 3, hydrogen will be generally used, but the isotopic effect will also be addressed by performing experiments and investigating the beam optics with deuterium.

Table 4 Diagnostic systems and basic software required for first SPIDER operation phase.

\begin{tabular}{|c|c|c|}
\hline Stage & Diagnostics & Outputs from basic analysis programmes \\
\hline \multirow{3}{*}{1} & source thermocouples & distribution of heat deposited over source surfaces \\
\hline & $\begin{array}{c}\text { source emission spectroscopy } \\
\text { (spectra and line emission evolution) }\end{array}$ & $\begin{array}{l}\text { emission spectra along different LoS vs time; } \\
\text { visualisation of plasma inhomogeneity vs time }\end{array}$ \\
\hline & basic electrostatic probes & $\begin{array}{l}\text { distribution of electric signals over plasma grid vs } \\
\text { time }\end{array}$ \\
\hline \multirow{4}{*}{2} & STRIKE & beam power distribution among beamlet groups \\
\hline & beam emission spectroscopy & $\begin{array}{l}\text { emission spectra along different LoS vs time; } \\
\text { visualisation of beam inhomogeneity vs time }\end{array}$ \\
\hline & beam dump thermocouples & visualisation of beam inhomogeneity \\
\hline & IR thermography & beam power distribution over beam dump vs time \\
\hline 3 & $\begin{array}{l}\text { completion of the whole set of } \\
\text { electrostatic probes, source } \\
\text { spectroscopy and beam emission } \\
\text { spectroscopy }\end{array}$ & \\
\hline \multirow{2}{*}{4} & laser absorption spectroscopy & amount of caesium in source vs time \\
\hline & cavity ring down spectroscopy & amount of $\mathrm{H}^{-} / \mathrm{D}^{-}$near plasma grid vs time \\
\hline
\end{tabular}

Table 4 gives a summary of the diagnostics required before each operational stage starts; in the diagnostics composed of multi-sensors, a subset of the system is sufficient at the beginning and the system can be completed during the third stage.

After the exploitation of the early experimental phase, the RF power will be increased and operation with deuterium will be also performed and continued toward the full achievement of the whole set of requirements.

A tentative operational sequence under analysis for the production of the accelerated beam of negative ions is sketched in Figure 8. Before the start of the discharge, the ignition filaments are switched on and biased and the gas is injected into the source in order to prepare the seed electrons for subsequent ionisation of the gas in the source; application of RF power guarantees the generation of an inductive plasma. After plasma ignition the RF power is gradually raised to its target value. At the same time, the boundary conditions of the plasma are set up, using the power supplies for: bias of the plasma grid, polarization of the bias plate, current for the magnetic filter. Finally the extraction voltage and the acceleration voltage are gradually applied and the beam is produced. Similarly at the end of the beam pulse the high voltages are turned off before quenching the plasma. 


\section{Conclusions}

The realization of SPIDER, the full-size prototype of the RFdriven negative ion source for ITER NBI, is well advanced and the integration and testing of its main components is now in progress. The implementation of diagnostics and control systems is well advanced and the operation and scientific plan, based on a staged strategy, has already been outlined. The SPIDER first experimental phase will start in early 2018 , after the delivery and installation of the beam source; therefore several years of operation are expected before the use of the heating NBIs in ITER. This significant experimental period will be dedicated to accomplish the SPIDER mission: to achieve the ITER target requirements in a radiofrequencydriven device having the same characteristics and size as the ITER NBI negative ion source and to improve its performance in terms of reliability and availability, thus assuring a significant risk mitigation for the successful operation in ITER.

\section{Acknowledgements}

The work leading to this publication has been funded partially by Fusion for Energy. This publication reflects the views only of the authors, and F4E cannot be held responsible for any use which may be made of the information contained therein. The views and opinions expressed herein do not necessarily reflect those of the ITER Organization.

\section{References}

[1] R. Hemsworth et al., Rev. Sci. Instrum. 79 (2008) 02C109

[2] V. Toigo et al., Nucl. Fusion 55 (2015) 083025

[3] V. Toigo et al., Fusion Eng. Des., DOI: 10.1016/j.fusengdes.2016.11.007

[4] E. Speth et al., 2006 Nucl. Fusion 46 S220

[5] U. Fantz et al., Rev. Sci. Instrum. 87 (2016) 02B307

[6] N. Pilan et al., poster contribution at 29th SOFT (Prague 2016)

[7] M. De Muri et al., poster contribution at 29th SOFT (Prague 2016)

[8] R. Pasqualotto et al., Rev. Sci. Instrum. 83 (2012) 02B103

[9] R. Pasqualotto et al., submitted to AIP conference Proceedings

[10] M. Cavenago t al., Rev. Sci. Instrum. 87 (2016) 02B320 
[11] U. Fantz et al., Nucl. Fusion 49 (2009) 125007

[12] K. Tsumori et al., Rev. Sci. Instrum. 83 (2012) 02B116

[13] K. Ikeda, H. Nakano, K. Tsumori et al., New J. Phys. 15 (2013) 103026

[14] http://www.mdsplus.org/

[15] G. Serianni et al., Rev. Sci. Instrum. 87 (2016) 02B927

[16] P. Franzen et al., Fusion Eng. Des. 88 (2013) 3132

[17] P. Agostinetti et al., Nucl. Fusion 51 (2011) 063004

[18] P. Agostinetti et al., Nucl. Fusion 56 (2016) 016015 